\title{
Microscopic Detection of Mixed Malarial Infections: Improvement by Saponin Hemolysis
}

\author{
Augustine U. Orjih Preethi Cherian Suad AlFadhli \\ Department of Medical Laboratory Sciences, Faculty of Allied Health Sciences, Kuwait University, Kuwait
}

\section{Key Words}

Malaria $\cdot$ Mixed infection $\cdot$ Saponin $\cdot$ Culture enrichment

\begin{abstract}
Objective: The objective of the present study was to determine whether saponin hemolysis could improve microscopic detection of malaria parasites in human blood, since it has been previously reported that the technique has been used to enrich Plasmodium falciparum culture to $\geq 90 \%$ parasitemia. Material and Methods: Blood samples from suspected malaria cases were first examined in routine thick and thin smears under the microscope. The sample $(1 \mathrm{ml})$ was then hemolyzed with $0.015 \%$ saponin in saline and centrifuged, the separated pellet was stained with Giemsa stain and examined microscopically, using PCR to confirm species identification. Results: With P. falciparum in vitro culture, the proportions of infected erythrocytes were approximately $0.7-2 \%$ before and $65-97 \%$ after saponin hemolysis, confirming published reports. In contrast, there was little or no increase in the proportions of intact infected erythrocytes after saponin hemolysis of clinical blood specimens. However, 20-600 hemolyzed parasites were detected per field under the microscope after saponin hemolysis of patients' blood samples that contained only 1-15 parasites per field in conventional thick smears. In addition, more $P$. falciparum gametocytes were detected after saponin hemolysis. Con-
\end{abstract}

clusion: Saponin hemolysis concentrated the parasites in large volumes of blood into a small volume that could be smeared on a slide. This concentration method made it easy to detect malaria parasites and reduced the time needed for microscopy. In the present study, the method was comparable to $P C R$ for the identification of $P$. vivax and $P$. falciparum mixed infections.

Copyright $\odot 2008$ S. Karger AG, Basel

\section{Introduction}

In addition to estimated mortality rate of approximately 12 per 1,000 children in some endemic areas [1], malaria has become a worldwide health problem largely because of continuously increasing human migration. Millions of people originally from Africa, Asia and the Americas, where different Plasmodium species are endemic, now live in North America and Europe, where there is no malarial transmission [2]. Although some of these migrants have become naturalized citizens of the USA, the European Union and Canada, they still visit their home countries either for business or nostalgia. The oil-rich countries in the Arabian Gulf region are heavily populated by expatriate workers from the Indian subcontinent; thus, making imported malaria a major health problem in nonendemic areas such as Kuwait [3]. Tour-

\section{KARGER}

Fax +4161306 1234 E-Mail karger@karger.ch www.karger.com
(C) 2008 S. Karger AG, Basel

$1011-7571 / 08 / 0176-0458 \$ 24.50 / 0$

Accessible online at:

www.karger.com/mpp
Augustine U. Orjih

Department of Medical Laboratory Sciences

Faculty of Allied Health Sciences, Kuwait University

PO Box 31470, 90805 Sulaibikhat (Kuwait)

Tel./Fax +965 482 4799, E-Mail orjih@hsc.edu.kw 
ism to malaria-endemic areas by Americans and Europeans continues to increase, and the travelers need to be checked for malarial infection upon return to the nonendemic areas, particularly if they wish to donate blood for transfusion purposes [4].

Recent efforts to control malaria include the continued search for reliable diagnostic techniques. The conventional thick and thin blood smear technique is reliable when it detects malaria parasites, but is less reliable if no infected erythrocyte is detected. Included in the criticisms are that only 1-3 drops, equivalent to 5-30 $\mu$ l, of blood are usually examined, and that it requires experienced microscopists who must have the patience to spend up to 30 min carefully examining 1 slide, if necessary. Some investigators have modified the staining technique to reduce the time, without addressing the need to examine larger quantities of blood $[5,6]$.

Among the nonmorphological methods for malaria detection, the antigen capture methods $[7,8]$ are less time-consuming and probably require limited experience; but they are not very sensitive or specific $[9,10]$. An immunofluorescent antibody test requires an expensive microscope, and other serological techniques require reagents that may not be readily available in developing countries. Immunological tests can provide information about prior exposure to malaria, but may not differentiate between past and current infections. The polymerase chain reaction (PCR) is an indispensable research tool in molecular biology, and is also widely used for diagnosis [11]. Its biggest advantage is reliable species identification. However, the required instruments and reagents are expensive and it is more time-consuming than microscopic examination of slides.

Our current research is focused on increasing the reliability of microscopic diagnoses of malaria, including double infections. It has been found, using a laboratory strain, that intact $P$. falciparum ring-infected erythrocytes could be isolated from the culture by controlled hemolysis with $0.015 \%$ saponin solution $[12,13]$. In the current research, we investigate the effect of this technique on blood specimens from malaria patients.

\section{Materials and Methods}

\section{Blood Specimens}

Blood samples were collected from local hospital laboratories within a day after they were used for routine malaria tests, which involved microscopic examination of Giemsa-stained thick and thin blood smears. The samples were in tubes containing EDTA as the anti-coagulant, and their volume usually ranged from ap- proximately $1.5-4 \mathrm{ml}$. The patients were mostly adult males from the Indian subcontinent, some parts of Africa and the Middle East, where malaria is endemic. They were outpatients, and were examined for malaria because they were from endemic areas and had fever of unknown origin. Information on other possible illnesses and laboratory data were not available. Prior to saponin hemolysis, thick and thin smears were prepared and examined microscopically in our research laboratory. The research project was approved by the Ethical Committee, Health Sciences Centre, Kuwait University.

\section{Saponin Hemolysis}

Saponin powder (Sigma) was dissolved in physiological saline, $\mathrm{pH} 7.4$, to obtain a $0.015 \%$ solution. It was prepared every day the test was performed, and kept refrigerated or on ice when not in use. Unless stated otherwise, all centrifugation procedures in this study was done at 1,600 $\mathrm{g}$, using a bench-top centrifuge. First, $1 \mathrm{ml}$ of the test blood sample was transferred to a sterile $15-\mathrm{ml}$ plastic centrifuge tube with a screw cap, and centrifuged for 10 min to remove the plasma. The pellet, which contained both erythrocytes and leukocytes, was washed twice with $5 \mathrm{ml}$ of sterile saline. The volume of the pellet after the washing was usually $0.3-0.6 \mathrm{ml}$. It was loosened by gentle agitation before $4 \mathrm{ml}$ of cold saponin solution was added to the tube, and quickly mixed by inverting and tapping at the bottom of the tube repeatedly until all the cells had been suspended. The tube was then placed on ice and allowed to stand for $30 \mathrm{~min}$, mixing occasionally to keep the cells in suspension, and at the same time monitor the progress of hemolysis. After $30 \mathrm{~min}$, the tube was centrifuged for $5 \mathrm{~min}$ and the supernatant was quickly transferred to another sterile centrifuge tube. The pellet was suspended again in $2-5 \mathrm{ml}$ of saponin solution, depending on the quantity of the cell pellet, and incubated on ice for 5-15 $\mathrm{min}$. The suspension was centrifuged for $5 \mathrm{~min}$ to obtain pellet A. The supernatant was transferred to the tube containing the first supernatant and centrifuged for $30 \mathrm{~min}$ to obtain pellet B. P. falciparum cultures in vitro were enriched as described previously [12].

\section{Preparation of Smears for Microscopic Examination}

Thin smears of patients' blood, pellet A and in vitro cultures were prepared on glass slides and allowed to dry at room temperature. Thick smears of patients' blood before saponin, as well as pellet $B$ after saponin hemolysis were prepared on an area of $2 \mathrm{~cm}$ diameter and containing $10 \mu \mathrm{l}$ of the blood or pellet B on glass slides, which were allowed to dry at room temperature. Before staining for $30 \mathrm{~min}$ with $10 \%$ Giemsa stain, the slides, except the thick blood smears, were fixed with methanol.

\section{Polymerase Chain Reaction}

Saponin-isolated cell pellet was suspended in $300 \mu \mathrm{l}$ of distilled water. The suspension was transferred into a $1.5-\mathrm{ml} \mathrm{mi-}$ crofuge tube and boiled for $10 \mathrm{~min}$, after which it was centrifuged in a microfuge for $10 \mathrm{~min}$ and the supernatant was used for PCR assay. P. malariae and P. ovale are rarely seen in Kuwait; therefore, the PCR tests were limited to P. vivax and P. falciparum. The species-specific primer pairs described by Morassin et al. [14] were used, including 5' -CAC CAT TAA GTA CAT CAC-3' and 5'-TGT TAA TAC AAC TCC AAT-3' for $P$. vivax, and $5^{\prime}$-GGA ATG TTA TTG CTA ACA C-3' and 5'-AAT GAA GAG CTG TGT ATC-3' for P. falciparum. Strain-specific PCR was also done on a few 
P. falciparum isolates, using the MSP-2 primer pair 5'-GAG TAT AAG GAG AAG TAT GG-3' and 5'-CCT GTA CCT TTA TTC TCT GG-3' [15].

The PCR was done in essentially the same way described by Wooden et al. [15]. Briefly, $3 \mu \mathrm{l}$ of DNA extract was mixed with $2.5 \mathrm{ml}$ of $\times 10$ PCR buffer containing $15 \mathrm{mM}$ of $\mathrm{MgCl}_{2}$ (Applied Biosystems), $4 \mu \mathrm{M}$ each of forward and reverse primers, $2.5 \mathrm{mM}$ of dNTP and $0.25 \mathrm{U}$ of Taq polymerase (Applied Biosystems). The mixture was filled to $22 \mu \mathrm{l}$ with distilled water. In most cases, DNA amplification was carried out in 40 cycles, using the Gene Amp PCR System 9700 Thermocycler. The amplified product was loaded onto a $1.5 \%$ agarose gel and the electrophoresis ran for 30 $\mathrm{min}$. The bands were observed and photographed using a gel documentation system and the appropriate computer software.

\section{Results}

\section{Parasitemia Levels before Saponin Hemolysis}

The total numbers of $P$. falciparum and $P$. vivax cases, based on hospital diagnoses, were 27 and 55, respectively. We observed that in the whole blood the parasitemia levels of $P$. falciparum were $0.2-4 \%$, except 1 specimen that contained $19 \%$ parasitemia. Of the $P$. vivax cases, 46 had parasitemia levels under $0.8 \%$, whereas 9 had $1-3 \%$. An additional 8 blood samples were classified by the hospitals as mixed infections of $P$. falciparum and $P$. vivax.

\section{Proportion of P. falciparum-Infected Erythrocytes after Saponin Hemolysis}

This part of the result considered ring-infected erythrocytes that remained intact before and after exposure to saponin. In the experiments with blood samples from 27 falciparum malaria patients, it was found that the mean proportions of intact ring-infected erythrocytes in pellet A after saponin hemolysis were not significantly higher than the parasitemia in the original blood samples. Large quantities of hemolyzed parasites were observed in pellet B (see Materials and Methods). Depending on the initial parasitemia, the number of parasites per field under a $\times 100$ oil immersion objective ranged from 20 to 600 . For comparison, the direct thick smears prepared from the same blood samples contained easily identifiable parasites, but in the order of 1-50 per field.

\section{Proportion of P. vivax-Infected Erythrocytes after}

Saponin Hemolysis

Peripheral blood from a $P$. vivax infection usually contains ring forms, trophozoites, schizonts and gametocytes of the parasite. After the saponin treatment described here, it was found that virtually all the different forms of the parasite were hemolyzed (fig. 1).

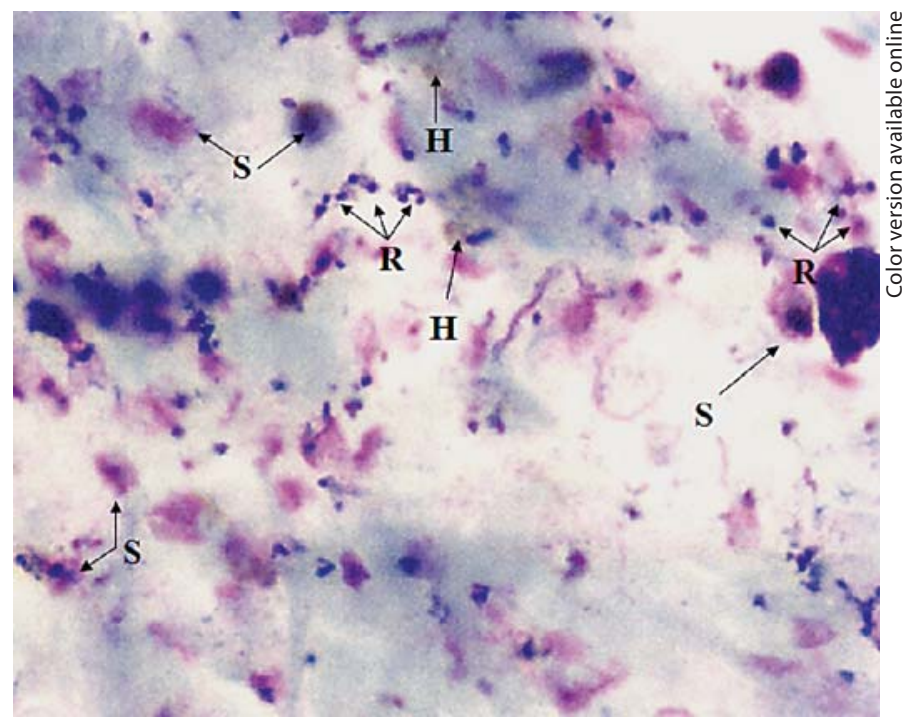

Fig. 1. $P$. vivax after saponin hemolysis. Initial parasitemia was $0.07 \%$, including ring forms (R), schizonts (S) and hemozoin debris $(\mathrm{H})$.

Composition of P. vivax and P. falciparum Mixed Infections after Saponin Hemolysis

Here, we use 1 case to illustrate how saponin hemolysis has helped to microscopically identify an unusual intra-erythrocytic mixed $P$. vivax and $P$. falciparum infection. A 35-year-old man arrived in Kuwait from India and was diagnosed in a routine examination as having only vivax malaria. Giemsa-stained thin blood smears contained easily detectable $P$. vivax at all developmental stages, but there were also some appliqué ring forms and some with double chromatin dots (not shown). There were some $P$. vivax trophozoites that also contained tiny ring forms, possibly P. falciparum (fig. $2 \mathrm{a}-\mathrm{c}$ ), as well as infected erythrocytes possibly containing the ring forms of both P. falciparum and P. vivax (fig. 2d-f). P. falciparum gametocy tes were not detected in the regular thick and thin smears. Giemsa-stained pellet smears obtained after saponin hemolysis contained 2 populations of ring forms, one stained deep blue and the other stained purple, raising the possibility that the ring forms of the 2 species might have stained differently in color. However, the clear proof of mixed infection was the presence of $P$. falciparum gametocytes detected only after saponin hemolysis (fig. 3), and PCR results showing P. vivax and P. falciparum DNA amplification products.

All the mixed infections identified in the present study after saponin hemolysis were based on detection of $P$. fal- 
Fig. 2. Invasion of the same erythrocyte by $P$. vivax $(\mathrm{Pv})$ and $P$. falciparum $(\mathrm{Pf})$. a-c Erythrocytes containing $P$. vivax trophozoites and tiny ring forms that could be $P$. falciparum. d In addition to the $P$. vivax gametocyte (upper left), there is 1 erythrocyte containing 2 blue-stained ring forms, possibly $P$. falciparum together with a larger ring (possibly $P$. vivax purplecolored nucleus). e The erythrocyte containing 2 rings is enlarged, indicating $P$. vivax; however, the smaller ring is probably $P$. falciparum. $f$ The multiply infected erythrocyte contains a ring with double chromatin dots and another appliqué form; however, it appears somewhat enlarged and has stained purple, more like erythrocytes containing $P$. vivax than those containing P. falciparum.

Fig. 3. Identification of mixed malaria infections. After saponin hemolysis, the blood sample was centrifuged for $5 \mathrm{~min}$ to obtain a pellet (a) and the supernatant was centrifuged again for 30 min to obtain another pellet (b). Detection of P. falciparum gametocytes (Pfg) provided the definitive evidence that the case was a mixed infection, even though $P$. vivax was predominant. $\mathrm{Pvg}=P$. vivax gametocytes; $\mathrm{Pvt}=$ $P$. vivax trophozoite; $\mathrm{Pvs}=P$. vivax schizonts; $\mathrm{R}=$ ring.
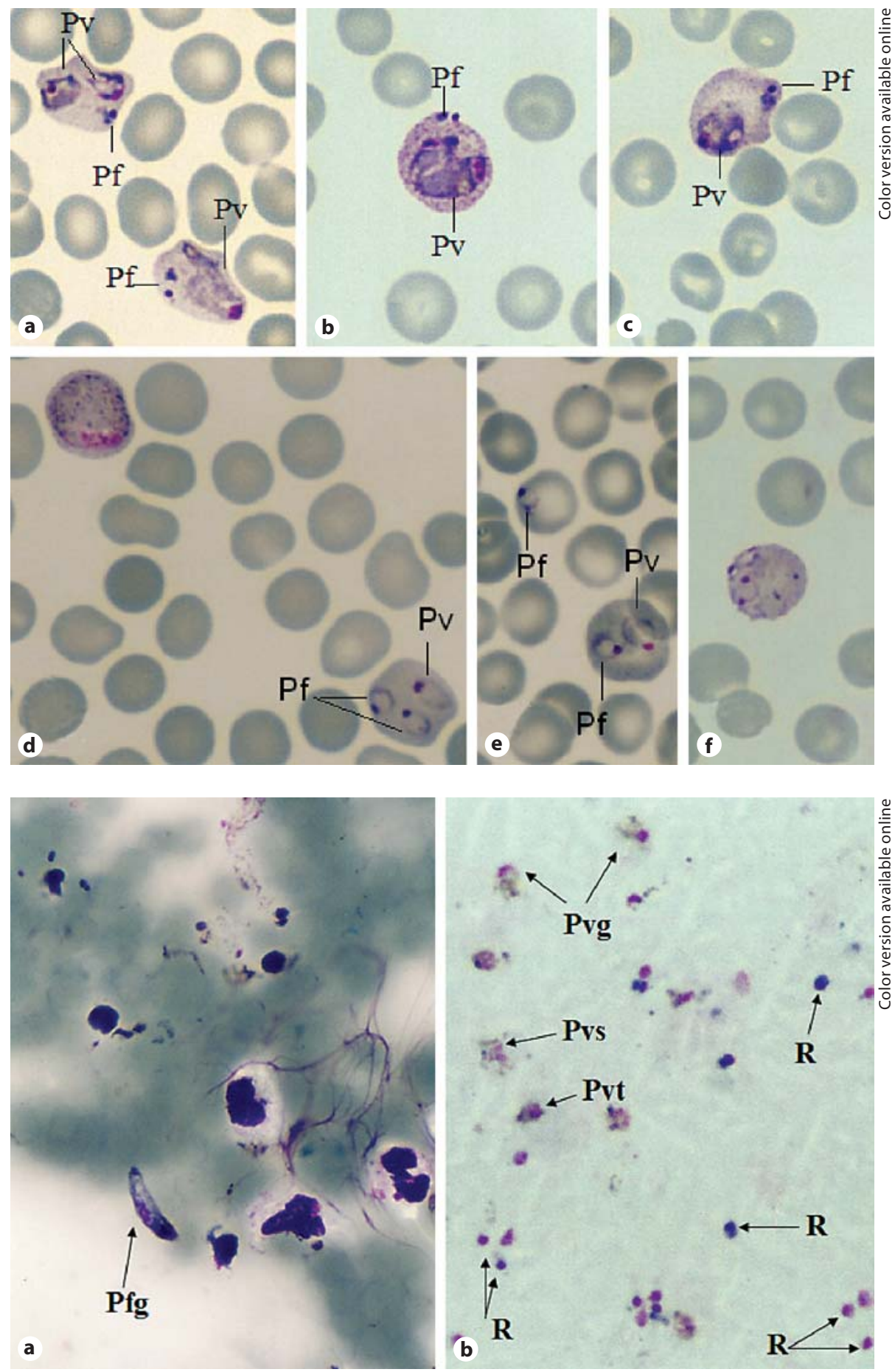

ciparum gametocytes, together with trophozoites and gametocytes of $P$. vivax, and were confirmed by PCR. The method confirmed all of the 8 mixed infections diagnosed in routine thick and thin smears, but detected 11 additional mixed infections among those that were originally diagnosed as either $P$. vivax or $P$. falciparum alone (table 1). Furthermore, saponin hemolysis identified $P$. falciparum in 2 of the 31 blood samples from individuals that were considered malaria-free in routine examination. Prior to saponin hemolysis, the 31 blood samples were microscopically diagnosed malaria-free by both hospital technologists and the principal investigator. 
Table 1. Malaria detection by microscopy before and after saponin hemolysis compared to PCR

\begin{tabular}{llll}
\hline Malaria diagnosis & \multicolumn{2}{l}{ Parasitemia } & \\
\cline { 2 - 4 } & $\begin{array}{l}\text { standard thick } \\
\text { and thin smears }\end{array}$ & $\begin{array}{l}\text { after saponin } \\
\text { hemolysis }\end{array}$ & PCR \\
\hline$P$ vivax (55 cases) & 55 & $55(9 \mathrm{MI})$ & $55(9 \mathrm{MI})$ \\
$P$. falciparum (27 cases) & 27 & $27(2 \mathrm{MI})$ & $27(2 \mathrm{MI})$ \\
Mixed infections (8 cases) & 8 & 19 & 19 \\
Negative control (31 cases) & 0 & $2^{1}$ & $2^{1}$
\end{tabular}

The blood specimens were examined for routine malaria diagnosis by experienced technologists prior to use in saponin hemolysis. Thick and thin smears were prepared from the blood samples immediately after the specimens were received for routine malaria examination. Parasites were detected in both thick and thin smears. MI = Mixed infections.

${ }^{1}$ P. falciparum.

\section{Discussion}

It was previously shown that $0.015 \%$ saponin solution selectively hemolyzed uninfected erythrocytes in synchronized $P$. falciparum culture, such that initial parasitemia of $<1 \%$ was increased to $75-99 \%$ [12]. It was hoped that the method could also be used to enrich intact infected erythrocytes from the blood of malaria patients, which would not only facilitate detection of hemolyzed parasites, but also retain them in intact erythrocytes and permit morphological characterization of both the parasites and host erythrocytes. Species-specific properties, such as enlargement of infected erythrocytes and production of Schüffner's dots and Maurer's clefts, are only easy to observe in intact erythrocytes. Unfortunately, the technique did not work as well with clinical specimens as it did with in vitro cultures. The mechanism of saponin resistance by $P$. falciparum-infected erythrocytes is unclear, but it is known that saponin binds to membrane cholesterol, and erythrocytes with decreased levels of membrane cholesterol were partially resistant to saponin destruction $[16,17]$.

Careful examination of the pellets recovered after saponin hemolysis of clinical blood specimens yielded other results that should facilitate detection of malaria parasitemia, improve identification of mixed $P$. falciparum and $P$. vivax infections and reduce the length of time spent on microscopy. Because $P$. falciparum gametocytes remained morphologically intact, and the parasites concentrated from relatively large quantities of blood could be smeared on 1 slide for microscopic examination, even the small numbers of gametocytes that could not be detected in regular thick and thin smears could be detected after saponin hemolysis. Here, 9 out of 55 (approximately 16\%) malaria cases, which were originally only diagnosed as $P$. vivax infections, were found after saponin hemolysis to also contain P. falciparum, and it was noteworthy that microscopic examination of saponin-concentrated pellets produced results comparable to the PCR test (table 1). Future studies with large numbers of specimens will be needed to confirm whether the saponin hemolysis technique described here will continue to produce morphological results comparable to plasmodia DNA detection by PCR.

While gametocyte shape is not the only morphological characteristic that differentiates $P$. falciparum from other Plasmodium species, it is perhaps the most definitive and easily recognizable marker. The other differential properties such as multiple invasions of erythrocytes, double chromatin dots and localization of the parasites at the periphery of the infected erythrocytes can also be found in $P$. vivax, albeit less frequently [18].

The modified saponin concentration technique described here also concentrated white blood cells, among them neutrophils and monocytes that contained phagocytosed $P$. vivax parasites. The relatively healthy appearance of the gametocytes suggested that the phagocytosis might have happened in vitro after the blood had been drawn from the patients.

Like the other methods for malaria detection, saponin hemolysis may be criticized in a number of ways: it requires relatively large quantities of blood to yield optimal results, and, therefore, may not be suitable for infants and small children. The preparation takes a longer time than making regular thick and thin smears and it requires a centrifuge that may not be available in poorly equipped laboratories. The importance of well-stained thin smears is clearly evident in this report, where mixed invasions of 1 erythrocyte by $P$. vivax and P. falciparum were detected, perhaps for the first time.

\section{Conclusion}

The saponin hemolysis technique described here was initially developed for elimination of uninfected erythrocytes from in vitro $P$. falciparum cultures for research purposes. The present work indicates that the method could also be useful in malaria diagnosis, as it facilitates detection of mixed infections and hemolyzed parasites.

\section{Acknowledgment}

This work was supported by Kuwait University, Research Grant No. NM 01/02. 


\section{References}

-1 Morenromp EL, Williams BG, Gouws E, Dye C, Snow RW: Measurement of trends in childhood malaria mortality in Africa: an assessment of progress toward targets based on verbal autopsy. Lancet Infect Dis 2003;3: 349-358.

2 Greenwood B, Mutabingwa T: Malaria in 2002. Nature 2002;415:670-672.

3 Iqbal J, Hira PR, Al-Ali F, Sher A: Imported malaria in Kuwait (1985-2000). J Travel Med 2003;10:24-29.

$\checkmark 4$ Kitchen AD, Barbara JA, Hewitt PE: Documented cases of post-transfusion malaria occurring in England: a review in relation to current and proposed donor-selection guidelines. Vox Sang 2005;89:77-80.

$\checkmark 5$ Li A, Mansoor AH, Tan KSW, Lim CT: Observations on the internal and surface morphology of malaria infected blood cells using optical and atomic force microscopy. J Microbiol Methods 2006;66:434-439.

-6 Iqbal J, Hira PR, Al-Ali F, Khalid N, Sher A: Modified Giemsa staining for rapid diagnosis of malaria infection. Med Princ Pract 2003;12:156-159.
7 Cropley IM, Lockwood DNJ, Mack D, Pasvol G, Davidson RN: Rapid diagnosis of falciparum malaria by using ParaSight F test in travelers returning to the United Kingdom: prospective study. BMJ 2000;321:484-485

$>8$ Tham JM, Lee SH, Tan TM, Ting RC, Kara UA: Detection and species determination by PCR: comparison with microscopy and with Parasight-F and ICT malaria Pf tests in a clinical environment. J Clin Microbiol 1999; 37:1269-1273.

$\checkmark 9$ Mason DP, Kawamoto F, Lin K, Laoboonchai A, Wongsrichanalai C: A comparison of two rapid field immunochromatographic tests to expert microscopy in the diagnosis of malaria. Acta Trop 2002;82:51-59.

10 Bartoloni A, Sabatinelli G, Benucci M: Performance of two rapid tests for Plasmodium falciparum malaria in patients with rheumatoid factors. N Engl J Med 1998;338:1075.

$>11$ Johnston SP, Pieniazek NJ, Xayavong MV, Slemenda SB, Wilkins PP, da Silva AJ: PCR as a confirmatory technique for laboratory diagnosis of malaria. J Clin Microbiol 2006; 44:1087-1089.

12 Orjih AU: Saponin hemolysis for increasing concentration of Plasmodium falciparum infected erythrocytes. Lancet 1994;343:295.
$>13$ Orjih AU: Comparison of Plasmodium falciparum growth in sickle cells in low oxygen environment and candle-jar. Acta Trop 2005;94:25-34.

14 Morassin B, Fabre R, Berry A, Magnaval JF: One year's experience with the polymerase chain reaction as a routine method for the diagnosis of imported malaria. Am J Trop Med Hyg 2002;66:503-508.

15 Wooden J, Kyes S, Sibley CH: PCR and strain identification in Plasmodium falciparum. Parasitol Today 1993;9:303-305.

16 Bangham AD, Horne RW: Action of saponin on biological membranes. Nature 1962;196: 952-953.

17 Segal R, Milo-Goldstein I: The susceptibility of cholesterol-depleted erythrocytes to saponin-hemolysis. Biochim Biophys Acta 1978; 512:223-226.

18 Ash LR, Orihel TC: Atlas of Human Parasitology, ed 4. Chicago, ASCP Press, 1997, pp 145-151.

19 Bell D, Peeling RW, WHO Regional Office for the Western Pacific/TDR: Evaluation of rapid diagnostic tests: malaria. Nat $\mathrm{Rev} \mathrm{Mi-}$ crobiol 2006;4(9 suppl):S34-S38. 